\title{
A CLASSIFICATION OF IMMERSIONS OF THE TWO-SPHERE
}

\author{
BY \\ STEPHEN SMALE
}

An immersion of one $C^{1}$ differentiable manifold in another is a regular map (a $C^{1}$ map whose Jacobian is of maximum rank) of the first into the second. A homotopy of an immersion is called regular if at each stage it is regular and if the induced homotopy of the tangent bundle is continuous. Little is known about the general problem of classification of immersions under regular homotopy. Whitney [5] has shown that two immersions of a $k$-dimensional manifold in an $n$-dimensional manifold, $n \geqq 2 k+2$, are regularly homotopic if and only if they are homotopic. The Whitney-Graustein Theorem [4] classifies immersions of the circle $S^{1}$ in the plane $E^{2}$. In my thesis [3] this theorem is extended to the case where $E^{2}$ is replaced by any $C^{2}$ manifold $M^{n}, n>1$. As far as I know, these are the only known results. In this paper we give a classification of immersions of the 2 -sphere $S^{2}$ in Euclidean $n$-space $E^{n}, n>2$, with respect to regular homotopy.

Let $V_{n, 2}$ be the Stiefel manifold of all 2 -frames in $E^{n}$. If $f$ and $g$ are two immersions of $S^{2}$ in $E^{n}$, an invariant $\Omega(f, g) \in \pi_{2}\left(V_{n, 2}\right)$ is defined.

Theorem A. If $f$ and $g$ are $C^{2}$ immersions of $S^{2}$ in $E^{n}$, they are regularly homotopic if and only if $\Omega(f, g)=0$. Furthermore let $\Omega_{0} \in \pi_{2}\left(V_{n, 2}\right)$ and let a $C^{2}$ immersion $f: S^{2} \rightarrow E^{n}$ be given. Then there exists an immersion $g: S^{2} \rightarrow E^{n}$ such that $\Omega(f, g)=\Omega_{0}$. Thus there is a 1-1 correspondence between elements of $\pi_{2}\left(V_{n, 2}\right)$ and regular homotopy classes of immersions of $S^{2}$ in $E^{n}$.

Since $\pi_{2}\left(V_{3,2}\right)=0$, Theorem A implies:

Theorem B. Any two $C^{2}$ immersions of $S^{2}$ in $E^{3}$ are regularly homotopic.

That this should be so, is not obvious. For example, it is not trivial to see that a reflection of the unit sphere in $E^{3}$ is regularly homotopic to the identity on the unit sphere.

Since $\pi_{2}\left(V_{4,2}\right)=Z$, there are an infinite number of regular homotopy classes of $S^{2}$ in $E^{4}$. In fact we are able to obtain using results of [1],

Theorem C. Given $\gamma \in H^{2}\left(S^{2}\right), \gamma$ even, then there is an immersion of $S^{2}$ in $E^{4}$ such that the characteristic class of the normal bundle is $\gamma$. Furthermore, any two such $C^{2}$ immersions are regularly homotopic. There is no immersion of $S^{2}$ in $E^{4}$ with odd normal class.

In [2] it is proved for say $S^{2}$ in $E^{4}$ that the normal class of the immersion

Presented to the Society, February 23, 1957; received by the editors April 29, 1957. 
is twice the algebraic intersection number of Whitney. Hence two $C^{2}$ immersions of $S^{2}$ in $E^{4}$ (for which the algebraic intersection number is defined) are regularly homotopic if and only if they have the same algebraic intersection number. This answers a special case of a question of Whitney [6, p. 220].

Finally one can immediately obtain from Theorem A that two immersions of $S^{2}$ in $E^{n}, n>4$, are always regularly homotopic.

M. Hirsch, using the theory of this paper, has obtained a regular homotopy classification for closed 2-manifolds in $E^{n}, n>2$.

A slight extension of the methods in this paper yields a generalization of Theorem A to the case where $E^{n}$ is replaced by any $C^{2}$ manifold $M^{n}, n>2$. We state the results as follows.

If $M$ is a $C^{1}$ manifold, $F_{2}(M)$ denotes the bundle of 2 -frames of $M$. Let $N$ be a $C^{2}$ manifold of dimension greater than two, let $x_{0} \in F_{2}\left(S^{2}\right)$ and let $y_{0} \in F_{2}(N)$. An immersion $f: S^{2} \rightarrow N$ is said to be based at $y_{0}$ if $f_{*}\left(x_{0}\right)=y_{0}$ where $f_{*}: F_{2}\left(S^{2}\right) \rightarrow F_{2}(N)$ is induced by $f$. A regular homotopy is based at $y_{0}$ if every stage of it is. If $f$ and $g$ are two immersions of $S^{2}$ in $N$ based at $y_{0}$, then an invariant $\Omega(f, g) \in \pi_{2}\left(F_{2}(N), y_{0}\right)$ is defined.

THEOREM D. If $f$ and $g$ are as above, then they are regularly homotopic, with the homotopy based at $y_{0}$, if and only if $\Omega(f, g)=0$. Furthermore, let $\Omega_{0}$ $\in \pi_{2}\left(F_{2}(N), y_{0}\right)$ and let a $C^{2}$ immersion $f: S^{2} \rightarrow N$ based at $y_{0}$ be given. Then there exists an immersion $g: S^{2} \rightarrow N$ based at $y_{0}$ such that $\Omega(f, g)=\Omega_{0}$. Thus there is a 1-1 correspondence between elements of $\pi_{2}\left(F_{2}(N), y_{0}\right)$ and regular homotopy classes of $C^{2}$ immersions of $S^{2}$ in $N$ based at $y_{0}$.

The methods used in this paper are extensions of methods of [3]. It is to be hoped that these methods can be used to solve further questions on regular homotopy classes of immersions.

$\S 1$ is on fiber spaces in the sense of Serre. In $\$ 2$ a triple $(E, p, B)$ of function spaces is defined and shown to have the covering homotopy property (Theorem 2.1). To generalize 2.1 would be a big step in obtaining regular homotopy classification of higher dimensional spheres. In $\$ 3$ Theorem 2.1 is applied to compute the homotopy groups of the fiber $\Gamma_{c}$ of $(E, \pi, B)$ (or at least to reduce the computation to a topological problem). Finding the 0 th homotopy group of $\Gamma_{c}$ is roughly the solution of the local problem in the theory of 2-dimensional regular homotopy. In $\$ 4$, using the knowledge of $\pi_{0}\left(\Gamma_{c}\right)$, the main theorems stated in the Introduction (except Theorem D) are proved.

1. A triple $(E, p, B)$ consists of topological spaces $E$ and $B$ with a map $p$ from $E$ into $B$ (note $p$ is not necessarily onto). A triple has the CHP if it has the covering homotopy property in the sense of Serre. In that case we call $(E, p, B)$ or sometimes just $E$ a fiber space. The following was proved in [3]. 
Theorem 1.1. Suppose the triple $(E, p, B)$ has the CHP locally; that is, for each point $x \in B$, there exists a neighborhood $V$ of $x$ such that $\left(p^{-1}(V), p, V\right)$ has the CHP. Then $(E, p, B)$ has the CHP.

A homomorphism $\left(h, h^{\prime}\right)$ from a fiber space $(E, p, B)$ into a fiber space $\left(E^{\prime}, p^{\prime}, B^{\prime}\right)$ is a pair of maps $h: E \rightarrow E^{\prime}$ and $h^{\prime}: B \rightarrow B^{\prime}$ such that the following diagram commutes.

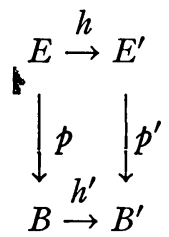

If $B$ and $B^{\prime}$ are the same space and $h^{\prime}$ the identity we will speak of $h$ as a homomorphism.

A map $f: X \rightarrow Y$ is a weak homotopy equivalence if (1) its restriction to each arcwise connected component of $X$ induces an isomorphism of homotopy groups and (2) it induces a 1-1 correspondence between the arcwise connected components of $X$ and $Y$.

Lemma 1.2. If $(E, p, B)$ is a fiber space then $p(E)$ consists of a set of arcwise connected components of $B$.

This follows immediately from the CHP.

Lemma 1.3. Let $\left(h, h^{\prime}\right)$ be a homomorphism from a fiber space $(E, p, B)$ to a fiber space $\left(E^{\prime}, p^{\prime}, B^{\prime}\right)$ such that $h$ and $h^{\prime}$ are both weak homotopy equivalences. Let $x_{0} \in B, y_{0}=h^{\prime}\left(x_{0}\right), F=p^{-1}\left(x_{0}\right)$ and $F^{\prime}=p^{\prime-1}\left(y_{0}\right)$. Then the restriction of $h$ to $F, \bar{h}: F \rightarrow F^{\prime}$ is a weak homotopy equivalence between $F$ and $F^{\prime}$.

This lemma is an immediate consequence of 1.2 , the exact homotopy sequence of a fiber space and the 5-lemma.

2. Let $D$ be the disk in the plane $E^{2}, D=\left\{(x, y) \mid x^{2}+y^{2} \leqq 1\right\}$ and $\dot{D}$ $=\left\{(x, y) \mid x^{2}+y^{2}=1\right\}$. However, unless specifying otherwise, we will refer to the points of $D$ with polar coordinates $(r, \theta)$ and use $\theta$ as the coordinate for $\dot{D}$. The points of Euclidean $n$-space $E^{n}$ will be considered to be vectors from some fixed origin.

A space $E$ is defined as the set of all regular maps of $D$ into $E^{n}$ (we always assume $n>2$ ) which satisfy the following condition. If $f \in E$ then the first derivatives of $f$ on the boundary of $D, \dot{D}$ are $C^{1}$ functions of the boundary variable $\theta$. The topology defined by the following metric is imposed on $E$.

$$
d(f, g)=\max \left\{d^{\prime}(f(p), g(p)), d^{\prime}\left(f_{x}(p), g_{x}(p)\right), d^{\prime}\left(f_{y}(p), g_{y}(p)\right) \mid p \in D\right\} .
$$

Here $d^{\prime}$ is the ordinary metric on $E^{n}$ and $f_{x}(p), f_{y}(p)$, etc., denote the obvious partial derivatives. In general we will call such a topology on a function space, the $C^{1}$ uniform topology. 
Let $V_{n, 2}$ be the Stiefel manifold of 2-frames (not necessarily orthonormal, but ordered, and independent pairs) in $E^{n}$. Let $V=V_{n, 2} \times E^{n}$ be all 2-frames at all points of $E^{n}$. Let $q$ be the projection of a frame onto its base point, $q: V \rightarrow E^{n}$, and $q_{1}, q_{2}$, the projections of a frame into its first and second vector respectively.

Let $B^{\prime}$ be the space of maps of $\dot{D}$ into $V$ with the compact open topology. Let $B$ be the subspace of $B^{\prime}$ satisfying the condition: If $f \in B$, then

(1) $q f(\theta)=\hat{f}(\theta)$ is regular,

(2) $\hat{f}^{\prime}(\theta)=q_{2} f(\theta)$, and

(3) $f$ is $C^{1}$.

A map $\pi: E \rightarrow B$ is defined as follows. If $f \in E$,

(1) $q \pi(f)(\theta)=f(1, \theta)$,

(2) $q_{1} \pi(f)(\theta)=f_{r}(1, \theta)$,

(3) $q_{2} \pi(f)(\theta)=f_{\theta}(1, \theta)$.

The subscripts $r$ and $\theta$ as here always denote the respective partial derivatives. From the definitions of the spaces, $E$ and $B$, it follows that $\pi$ is well defined and continuous. The purpose of this section is to prove the following theorem.

\section{Theorem 2.1. The triple $(E, \pi, B)$ has the CHP.}

Let $g \in B$ be given. We choose a neighborhood $U$ of $g$ in $B$ as follows: Let $A$ be the minimum angle in radians between $q_{1} g(\theta)$ and $q_{2} g(\theta)$ as $\theta$ ranges over $\dot{D}$. Let $L$ be the minimum of the magnitudes of $q_{1} g(\theta)$ and $q_{2} g(\theta)$ as $\theta$ ranges over $\dot{D}$. Choose $U$ such that for $h \in U$,

(1) the angle between $q_{i} h(\theta)$ and $q_{i} g(\theta)$ is $<A / 100$, and

(2) $\left|q_{i} h(\theta)-q_{i} g(\theta)\right|<A L / 100$ for $i=1,2$ and $\theta \in \dot{D}$. By the topology of $B, U$ can be chosen as above.

By 1.1. it is sufficient for the proof of 2.1 to show that $\left(\pi^{-1}(U), \pi, U\right)$ has the CHP. Let $h_{v}: P \rightarrow U$ be a given homotopy, $P$ a polyhedron, and $\bar{h}: P \rightarrow E$ a covering of $h_{0}$. We will construct a covering homotopy $\bar{h}_{v}: P \rightarrow E$. We may assume that $P$ is a cube (see, e.g. [3]).

A linear transformation of $E^{n}, Q_{v}(p)(\theta)$ is defined as follows. First, let $V_{v}(p)(\theta)$ be the plane defined by $q_{1} h_{0}(p)(\theta)$ and $q_{1} h_{v}(p)(\theta)$ (if it exists) and $\alpha_{v}(p)(\theta)$ the angle from the first to the second of these vectors. Then $Q_{v}^{*}(p)(\theta)$ is to be the rotation of $E^{n}$ which takes $V_{v}(p)(\theta)$ through the angle $\alpha_{v}(p)(\theta)$ and leaves the orthogonal complement fixed (if $V_{v}(p)(\theta)$ does not exist then $Q_{0}^{*}(p)(\theta)$ is the identity $\left.e\right)$. Finally $Q_{v}(p)(\theta)$ is $Q_{0}^{*}(p)(\theta)$ multiplied by the scalar $\left|q_{1} h_{v}(p)(\theta)\right| /\left|q_{1} h_{0}(p)(\theta)\right|$. Note that $\left[q_{1} h_{0}(p)(\theta)\right] Q_{v}(p)(\theta)=q_{1} h_{v}(p)(\theta)$ where $Q_{v}(p)(\theta)$ is to be considered as acting on the right. Also $Q_{v}(p)(\theta)$ is $C^{1}$ with respect to $\theta$ and continuous with respect to $v$ and $p$.

Lemma 2.2. Let $n>k>1, G_{n, k}$ be the Grassman manifold of oriented k-planes in $E^{n}$ and $S^{n-1}$ the unit vectors of $E^{n}$. Let a map $w: Q \rightarrow G_{n, k}$ be given which is 
homotopic to a constant where $Q$ is some polyhedron. Then there is a map $u: Q \rightarrow S^{n-1}$ such that for all $q \in Q, u(q)$ is perpendicular to the plane $w(q)$.

The proof may be adapted from the proof of 4.1 of [3] substituting $V_{n, k+1}$ for $V_{n, 2}$ in the proof.

Let $u: P \times I \times \dot{D} \rightarrow S^{n-1}$ be given by 2.2 , taking for $w: P \times I \times \dot{D} \rightarrow G_{n, 2}$ the map which sends $(p, v, \theta)$ into the plane of $E^{n}$ spanned by $q_{1} h_{v}(p)(\theta)$ and $q_{2} h_{v}(p)(\theta)$. Because $h_{0}(p)$ is covered by $\bar{h}$, one can prove that $w$ is homotopic to a constant map.

Now choose $\delta>0$ so that if $\left|v-v^{\prime}\right| \leqq \delta$ then

$$
\left|q h_{v}(p)-q h_{v^{\prime}}(p)\right|<\frac{A L}{1000 T}
$$

where $T=\max \left\{u_{\theta}(p, v, \theta) \mid p \in P, v \in I, \theta \in \dot{D}\right\}$.

Choose $r_{0}, 0 \leqq r_{0}<1$, such that for $p \in P, \theta \in \dot{D}$ and $r \geqq r_{0}$,

$$
\begin{aligned}
& \left|\bar{h}_{r}(p)(r, \theta)-\bar{h}_{r}(p)(1, \theta)\right|<(A / 100)\left|\bar{h}_{r}(p)(1, \theta)\right|, \\
& \left|\bar{h}_{\theta}(p)(r, \theta)-\bar{h}_{\theta}(p)(1, \theta)\right|<(A / 100)\left|\bar{h}_{\theta}(p)(1, \theta)\right|, \text { and } \\
& \frac{|\bar{h}(p)(r, \theta)-\bar{h}(p)(1, \theta)|}{1-r_{0}}<(101 / 100)\left|\bar{h}_{r}(p)(1, \theta)\right| \\
& |\bar{h}(p)(r, \theta)-\bar{h}(p)(1, \theta)|<\frac{A}{100 N}
\end{aligned}
$$

where $N=\max \left\{\left|Q_{v \theta}(p)(\theta)\right| \mid p \in P, v \in I, \theta \in \dot{D}\right\}$. That $r_{0}$ can be chosen satisfying (3) follows from the definition of the derivative $\bar{h}_{r}(p)(1, \theta)$.

Let $r_{1}=r_{0}+\left(1-r_{0}\right) / 200$ and choose a $C^{2}$ real function on $I=[0,1], \alpha(r)$, satisfying $\alpha(r)=0$ for $r \leqq r_{1}, \alpha(1)=1, \alpha^{\prime}(1)=0,|\alpha(r)| \leqq 1$, and such that $\left|\alpha^{\prime}(r)\right|<102 / 100\left(1-r_{0}\right)$. Let $\beta(r)$ be a real $C^{2}$ function on $I$ with $\beta(r)=0$ for $r \leqq r_{0}, \beta(1)=\beta^{\prime}(1)=0,|\beta(r)| \leqq 200$ and $\beta^{\prime}(r)>100 \alpha^{\prime}(r)$ for $r_{1} \leqq r \leqq 1$. The proofs that functions $\alpha(r)$ and $\beta(r)$ exist as above are not difficult and will be omitted.

Let

$$
M(v)=\max \left\{\left|q h_{v}(p)(\theta)-q h_{0}(p)(\theta)\right| \mid p \in P, \theta \in \dot{D}\right\} .
$$

Let

$$
\bar{Q}_{v}(p)(r, \theta)=e+\alpha(r)\left[Q_{v}(p)(\theta)-e\right]
$$

where $e$ is the identity transformation.

The covering homotopy $\bar{h}_{v}: P \rightarrow E$ is defined as follows for $v \leqq \delta$.

$$
\begin{aligned}
\bar{h}_{v}(p)(r, \theta)= & {[\bar{h}(p)(r, \theta)-\bar{h}(p)(1, \theta)] \bar{Q}_{v}(p)(r, \theta) } \\
& +\alpha(r)\left[q h_{v}(p)(\theta)-q h_{0}(p)(\theta)\right]+\beta(r) M(v) u(p, v, \theta)+\bar{h}(p)(1, \theta) .
\end{aligned}
$$

The following derivatives are easily computed. 


$$
\begin{gathered}
P_{r}=\bar{h}_{v r}(p)(r, \theta)=\bar{h}_{r}(p)(r, \theta) \bar{Q}_{v}(p)(r, \theta)+[\bar{h}(p)(r, \theta)-\bar{h}(p)(1, \theta)] \bar{Q}_{v r}(p)(r, \theta) \\
+\alpha^{\prime}(r)\left[q h_{v}(p)(\theta)-q h_{0}(p)(\theta)\right]+\beta^{\prime}(r) M(v) u(p, v, \theta) \\
P_{\theta}=\bar{h}_{v \theta}(p)(r, \theta)=\left[\bar{h}_{\theta}(p)(r, \theta)-\bar{h}_{\theta}(p)(1, \theta)\right] \bar{Q}_{v}(p)(r, \theta) \\
+[\bar{h}(p)(r, \theta)-\bar{h}(p)(1, \theta)] \bar{Q}_{v \theta}(p)(r, \theta)+\alpha(r)\left[q_{2} h_{v}(p)(\theta)-q_{2} h_{0}(p)(\theta)\right] \\
+\beta(r) M(v) u_{\theta}(p, v, \theta)+\bar{h}_{\theta}(p)(1, \theta) \\
\bar{Q}_{v r}(p)(r, \theta)=\alpha^{\prime}(r)\left[Q_{v}(p)(\theta)-e\right] \\
\bar{Q}_{v \theta}(p)(r, \theta)=\alpha(r) Q_{v \theta}(p)(\theta) .
\end{gathered}
$$

Then it can be checked that $\bar{h}_{v}(p)$ has the following properties.

(1) $\bar{h}_{v}(p)$ is $C^{1}$.

(2) $\vec{h}_{v}(p)$ has derivatives with respect to $\theta$ and $r, C^{1}$ with respect to $\theta$ on $\dot{D}$.

(3) $\bar{h}_{0}(p)(r, \theta)=\bar{h}(p)(r, \theta)$.

(4) $\bar{h}_{v}(p)(1, \theta)=q h_{v}(p)(\theta)$.

(5) $\bar{h}_{v r}(p)(1, \theta)=q_{1} h_{v}(p)(\theta)$.

We will show

(6) $\bar{h}_{v}(p)$ is regular.

To show (6) it is sufficient to show that the derivatives $P_{r}$ and $P_{\theta}$ are independent. From the various choices made it can be checked that $P_{r}$ is "close" to $\bar{h}_{r}(p)(1, \theta)+\beta^{\prime}(r) M(v) u(p, v, \theta)$ while $P_{\theta}$ is "close" to $\vec{h}_{\theta}(p)(1, \theta)$. From this statement it follows that $P_{r}$ and $P_{\theta}$ are independent.

From (1), (2) and (6) it follows that $\bar{h}_{v}(p)$ is really in $E$, from (3) that $\bar{h}_{v}$ is a homotopy of $\bar{h}$ and from (4) and (5) that $\bar{h}_{v}$ covers $h_{v}$. For $\delta \leqq v \leqq 2 \delta$ define $\bar{h}_{v}$ as before using $\bar{h}_{\delta}$ instead of $\bar{h}$. Iteration yields a covering homotopy $\bar{h}_{v}$ for all $v \in I$. This proves 2.1 .

3. Let $x_{0} \in V$, and let $B_{0}$ be the subspace of $B$ with the further condition that for $f \in B_{0}, f(0)=x_{0}$ (see the previous section for notation). Let $E_{0}=\pi^{-1}\left(B_{0}\right)$ and let the restriction of $\pi$ to $E_{0}$ still be denoted by $\pi$. Then from 2.1 we have the theorem.

Theorem 3.1. The triple $\left(E_{0}, \pi, B_{0}\right)$ has the CHP.

Let $E^{\prime}$ be the space of all maps of pairs $\left(D, p_{0}\right)$ into $\left(V, x_{0}\right)$ with the compact open topology where $p_{0}$ is the point of $D,(r, \theta)=(1,0)$. Let $B_{0}^{\prime}$ be the subspace of $B^{\prime}\left(B^{\prime}\right.$ as in $\left.\S 2\right)$ with the condition if $f \in B_{0}^{\prime}$ then $f(0)=x_{0}$. A map $\pi^{\prime}: E^{\prime} \rightarrow B_{0}^{\prime}$ is defined by restriction to $D$, i.e., if $f \in E^{\prime},(\pi f)(\theta)=f(1, \theta)$.

Lemma 3.2. The triple $\left(E^{\prime}, \pi^{\prime}, B_{0}^{\prime}\right)$ has the CHP and $E^{\prime}$ is contractible.

Proof. Let $h_{v}: P \rightarrow B_{0}^{\prime}$ be a given homotopy and $\bar{h}: P \rightarrow E^{\prime}$ cover $h_{0}$ where $P$ is some finite polyhedron. A covering homotopy $\bar{h}_{v}: P \rightarrow E^{\prime}$ is defined as follows:

$$
\begin{array}{ll}
\bar{h}_{v}(p)(r, \theta)=\bar{h}(p)((v+1) r, \theta) & 0 \leqq r \leqq 1 /(1+v), \\
\bar{h}_{v}(p)(r, \theta)=h_{r(1+v)-1}(p)(\theta) & 1 /(1+v) \leqq r \leqq 1 .
\end{array}
$$


It remains to show that $E^{\prime}$ is contractible. Let $h_{t}: D \rightarrow D$ be a strong deformation of $D$ into the point $p_{0} \in D$. Then define $H_{t}: E^{\prime} \rightarrow E^{\prime}$ by

$$
H_{t}(f)(p)=f\left(h_{t}(p)\right), \quad f \in E^{\prime}, p \in D .
$$

It is easily seen that $H_{t}$ contracts $E^{\prime}$ in to the constant map $f_{0}: D \rightarrow x_{0}$. This proves 3.2 .

Leмma 3.3. The homotopy groups of $E_{0}$ vanish, i.e., $\pi_{k}\left(E_{0}\right)=0, k \geqq 0$.

Proof. Let $f: S^{k} \rightarrow E_{0}$ be given, $k \geqq 0$. We will show that $f$ is homotopic to a constant map. Let $A$ be the angle between the two vectors of the frame $x_{0} \in V$ and let $L$ be the minimum of their magnitudes. Then there exists a neighborhood $U$ of $p_{0}$ of $D$ such that for $q$ and $q^{\prime}$ of $S^{k}$ we have

$$
\begin{gathered}
\left|f_{r}(q)(r, \theta)-f_{r}\left(q^{\prime}\right)(r, \theta)\right|<A L / 100 \\
\left|f_{\theta}(q)(r, \theta)-f_{\theta}\left(q^{\prime}\right)(r, \theta)\right|<A L / 100
\end{gathered}
$$

for all $(r, \theta) \in U$. The existence of such a $U$ implies that there is a homotopy $H_{v}: S^{k} \rightarrow E_{0},-1 \leqq v \leqq 0$, with $H_{-1}(q)=f(q)$ and such that (1) and (2) above are satisfied with $H_{0}$ replacing $f$ and $(r, \theta)$ ranging over $D$. Let $q_{0} \in S^{k}$ and $e=H_{0}\left(q_{0}\right) \in E_{0}$. Then define $H_{v}: S^{k} \rightarrow E_{0}, 0 \leqq v \leqq 1$ by

$$
H_{v}(q)=(1-v) H_{0}(q)+v e .
$$

It follows from the choice of $U$ that $H_{v}(q)$ is regular. Then it is easily seen that $H_{v}(p)$ is well defined and that $H_{1}(q)=e$. Thus $f$ is homotopic to a constant and hence 3.3 is proved.

Let $F^{\prime}$ be the space of maps of $I_{2 \pi}=[0,2 \pi]$ into $V$ starting at $x_{0}$, and let $p^{\prime}: F^{\prime} \rightarrow V$ be the map which sends a path onto its end point. It is well-known that $\left(F^{\prime}, p^{\prime}, V\right)$ has the $\mathrm{CHP}$ and that $F^{\prime}$ is contractible. Note also that the fiber $p^{\prime-1}\left(x_{0}\right)$ is the space $B_{0}^{\prime}$.

Denote by $F$ the subspace of $F^{\prime}$ which satisfies conditions (1)-(3) of $B$ in $\S 2$. Let $p^{\prime}$ restricted to $F$ be denoted by $p$. Then $B_{0}=p^{-1}\left(x_{0}\right)$.

Lemma 3.4. The triple $(F, p, V)$ has the CHP.

Proof. Let $h_{v}: P \rightarrow V$ be a given homotopy where $P$ is a cube and let $\bar{h}: P \rightarrow F$ be a covering of $h_{0}$. We will construct a covering homotopy $\bar{h}_{v}: P \rightarrow F$.

Let $\phi: V \rightarrow T$ be the map of $V$ into $T$, the bundle of nontrivial tangent vectors of $E^{n}$, which is defined by dropping the first of the pair of vectors from each frame. Clearly $V$ becomes a fiber bundle over $T$ this way.

Let $R$ be the space of regular curves of $E^{n}$ starting with point and derivative $\phi\left(x_{0}\right)$ and with the $C^{1}$ uniform topology. Let $\tau: R \rightarrow T$ be defined by $\tau(f)=\left(f^{\prime}(1), f(1)\right)$. Then $(R, \tau, T)$ has the CHP. For a detailed description of this theory, see [3]. In this paper, a regular curve differs slightly from those of [3], but the transition in the theory from one definition to the other may be made without trouble. If $g_{v}: P \rightarrow T$ is the composition $\phi h_{v}$, the 
above remarks imply that there is a homotopy $\bar{g}_{v}: P \rightarrow R$ such that (1) $\tau \bar{g}_{v}=g_{v}$ and (2) $\bar{g}_{0}=\phi \bar{h}$.

Define a map $H: P \times I_{2 \pi} \times I \rightarrow T$ by $I H(p, \theta, v)=\left(\bar{g}_{v \theta}(p)(\theta), \bar{g}_{v}(p)(\theta)\right)$ and let $X$ be the bundle induced by $H$ with respect to the bundle $V$ over $T$. Let $q: X \rightarrow V$ be the corresponding bundle map. Since $P \times I_{2 \pi} \times I$ is contractible $X$ will be trivial.

Let $A=P \times \dot{I}_{2 \pi} \times I \cup P \times I_{2 \pi} \times 0$ and define as follows a cross-section $s: A \rightarrow X$ thinking of $X \subset V \times P \times I_{2 \pi} \times I$.

$$
\begin{aligned}
s(p, 0, v) & =\left(x_{0}, p, 0, v\right), \\
s(p, 2 \pi, v) & =\left(h_{v}(p), p, 2 \pi, v\right), \\
s(p, \theta, 0) & =(\bar{h}(p)(\theta), p, \theta, 0) .
\end{aligned}
$$

Now we can extend $s$ to a cross-section, still denoted by $s$, of $P \times I_{2 \pi} \times I$ into $X$ so it is $C^{1}$ with respect to $\theta$. Let $\bar{h}_{v}(p)(\theta)=q s(p, \theta, v)$. It can be easily checked that $\bar{h}_{v}$ is our desired covering homotopy.

Lemma 3.5. The homotopy groups $\pi_{k}(F)$ vanish, $k \geqq 0$.

The proof of 3.5 is not difficult and will not be given. The method is a slight extension of the proof of 6.2 of [3].

A map $\phi: E_{0} \rightarrow E^{\prime}$ is defined by the following equations. For $f \in E_{0}$, let

$$
\begin{gathered}
q \phi(f)(x, y)=f(x, y), \\
q_{1} \phi(f)(x, y)=f_{x}(x, y), \\
q_{2} \phi(f)(x, y)=f_{y}(x, y) .
\end{gathered}
$$

Here obviously we are using rectangular coordinates for the disk $D$. The difficulty of using polar coordinates to define such a map $\phi$ are apparent.

We define a map $\psi_{0}: B_{0} \rightarrow B_{0}^{\prime}$ in such a way that the following diagram commutes.

$$
\begin{aligned}
& \sum_{0} \stackrel{\phi}{\longrightarrow} E^{\prime} \\
& \sum_{B_{0} \stackrel{\psi_{0}}{\longrightarrow} B_{0}^{\prime}} \boldsymbol{i}^{\prime} .
\end{aligned}
$$

We define first a map $\psi: F \rightarrow F^{\prime}$ as follows. For $f \in F$ let

$$
\begin{aligned}
q \psi(f)(\theta) & =f(\theta), \\
q_{1} \psi(f)(\theta) & =\cos \theta q_{1} f(\theta)-\sin \theta q_{2} f(\theta), \\
q_{2} \psi(f)(\theta) & =\sin \theta q_{1} f(\theta)+\cos \theta q_{2} f(\theta) .
\end{aligned}
$$

The map $\psi$ is based on formulae for transformation from coordinates $(r, \theta)$ 
to coordinates $(x, y)$. In fact, (5) and (6) come from the following equations for the boundary of an immersion $g: D \rightarrow E^{n}$.

$$
\begin{aligned}
& g_{x}=\cos \theta g_{r}-\sin \theta g_{\theta}, \\
& g_{y}=\sin \theta g_{r}+\cos \theta g_{\theta} .
\end{aligned}
$$

The map $\psi_{0}: B_{0} \rightarrow B_{0}^{\prime}$ is the restriction of $\psi$. It can be checked now that (3) commutes.

Lemma 3.6. The map $\psi_{0}: B_{0} \rightarrow B_{0}^{\prime}$ is a weak homotopy equivalence.

Consider the diagram,

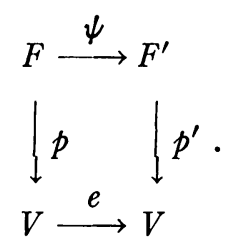

Here $e$ is the identity. It can be easily checked that (7) commutes in spite of the fact that $\psi$ is not the ordinary inclusion. Then by 1.3, 3.4 and 3.5, $\psi_{0}: B_{0} \rightarrow B_{0}^{\prime}$ is a weak homotopy equivalence.

Let $c \in B_{0}, \Gamma_{c}=\pi^{-1}(c)$, and $\pi^{\prime-1}(d)=\Omega_{d}$, where $d=\psi_{0}(c)$. Let $\phi_{0}: \Gamma_{c} \rightarrow \Omega_{d}$ be the restriction of $\phi$. Then from 1.3, 3.1, 3.2, 3.3 and 3.6 we obtain:

Theorem 3.7. The map $\phi_{0}: \Gamma_{c} \rightarrow \Omega_{d}$ is a weak homotopy equivalence.

From the exact homotopy sequences of the triples $\left(E^{\prime}, \pi^{\prime}, B_{0}^{\prime}\right)$ and $\left(F^{\prime}, p, V\right)$ (using 3.2) we obtain:

LEMmA 3.8. $\pi_{k}\left(\Omega_{d}\right)=\pi_{k+2}(V)=\pi_{k+2}\left(V_{n, 2}\right)$.

Combining 3.7 and 3.8 we have

Theorem 3.9. $\pi_{k}\left(\Gamma_{c}\right)=\pi_{k+2}\left(V_{n, 2}\right)$. In particular $\pi_{0}\left(\Gamma_{c}\right)=\pi_{2}\left(V_{n, 2}\right)$.

The last statement may be interpreted as giving the regular homotopy classes with fixed boundary conditions of a disk in $E^{n}$.

4. Let $f$ and $g$ be two $C^{2}$ immersions of $S^{2}$ in $E^{n}$. We can assume without loss of generality that they agree on a closed neighborhood $U$ of a point say $z_{0} \in S^{2}$. An invariant $\Omega(f, g) \in \pi_{2}\left(V_{n, 2}\right)$ is defined as follows. The space $D=S^{2}$-int $U$ is a topological disk, so we can assume there is a fixed field of 2 -frames defined over it. From this field $f$ and $g$ induce maps of $D$ into $V_{n, 2}$ which agree on the boundary $\dot{D}$ of $D$. Then by "reflecting" $g$ we obtain a map of the 2-sphere $S^{2}$ into $V_{n, 2}$. The homotopy class of this map clearly does not depend on the choices made. We denote this class by $\Omega(f, g) \in \pi_{2}\left(V_{n, 2}\right)$ (we can ignore the base point of $\pi_{2}\left(V_{n, 2}\right)$ because either $V_{n, 2}$ is simply connected or $\left.\pi_{2}\left(V_{n, 2}\right)=0\right)$. 
Theorem A of the Introduction is an immediate consequence of the preceding paragraph and 3.9.

Let $G_{n, 2}$ be the Grassman manifold of oriented 2-planes in $E^{n}$. An immersion $f: S^{2} \rightarrow E^{n}$ induces the tangential map $T_{f}: S^{2} \rightarrow G_{n, 2}$ by translating a tangent plane at a point of $f\left(S^{2}\right)$ to the origin of $E^{n}$. We denote by $\bar{T}_{f}$ the homotopy class of $T_{f}$, thus $\bar{T}_{f}$ is an element of $\pi_{2}\left(G_{n, 2}\right)$. Let $p: V_{n, 2} \rightarrow G_{n, 2}$ be the map which sends a frame into the plane it spans, and $p_{\sharp}: \pi_{2}\left(V_{n, 2}\right) \rightarrow \pi_{2}\left(G_{n, 2}\right)$ the induced homomorphism.

The next lemma follows from the definition of $\Omega(f, g)$.

Lemma 4.1. If $f$ and $g$ are two immersions of $S^{2}$ in $E^{n}$, then $p \sharp \Omega(f, g)=\bar{T}_{f}$ $-\bar{T}_{g}$.

We now prove Theorem $\mathrm{C}$ of the Introduction. Let $f$ and $g$ be two immersions of $S^{2}$ in $E^{4}$. Let $p_{*}: H_{2}\left(V_{4,2}\right) \rightarrow H_{2}\left(G_{4,2}\right)$ and $T_{f *}, T_{0^{*}}: H_{2}\left(S^{2}\right) \rightarrow H_{2}\left(G_{4,2}\right)$ be the homomorphisms induced by $p, T_{f}$, and $T_{g}$ respectively. Suppose that $S^{2}$ is oriented and that $s$ is the fundamental class of $H_{2}\left(S^{2}\right)$. Then by 4.1 and the Hurewicz theorem, $p_{*} \bar{\Omega}(f, g)=T_{f *}(s)-T_{g *}(s)$, where $\bar{\Omega}(f, g) \in H_{2}\left(V_{4,2}\right)$ corresponds to $\Omega(f, g)$ under the Hurewicz isomorphism. Let $\bar{W}(f)$ and $\bar{W}(g)$ be the normal classes belonging to $H^{2}\left(S^{2}\right)$ defined by the immersions $f$ and $g$ evaluated on $s$. Then by Chern-Spanier [1],

$$
T_{f *}(s)-T_{o *}(s)=2^{-1}[\bar{W}(f)-\bar{W}(g)] \phi
$$

where $\phi$ is a certain element of $H_{2}\left(G_{4,2}\right)$. Thus

$$
p_{*} \Omega(f, g)=2^{-1}[\bar{W}(f)-\bar{W}(g)] \phi
$$

and since $p_{*}$ is $1-1, \Omega(f, g)=0$ if and only if $\bar{W}(f)=\bar{W}(g)$. Then Theorem $\mathrm{C}$ follows from Theorem A.

Lastly we note an easy consequence of Theorem 2.1 .

Theorem 4.2. A regular map of $\dot{D}$ into $E^{n}, n>2$, can always be extended to a regular map of $D$ into $E^{n}$.

\section{REFERENCES}

1. S. S. Chern and E. Spanier, A theorem on orientable surfaces in four-dimensional space, Comment Math. Helv. vol. 25 (1951) pp. 1-5.

2. R. K. Lashof and S. Smale, On immersions of manifolds, to appear in Ann. of Math.

3. S. Smale, Regular curves on Riemannian manifolds, Trans. Amer. Math. Soc. vol. 87 (1958) pp. 492-512. 276-284.

4. H. Whitney, On regular closed curves in the plane, Compositio Math. vol. 4 (1937) pp.

5. - Differentiable manifolds, Ann. of Math. vol. 37 (1936) pp. 645-680.

6. - The self-intersections of a smooth $n$-manifold in $2 n$-space, Ann. of Math. vol. 45 (1944) pp. 220-246.

University of Chicago,

Chicago, Ill. 